\title{
Neurohypophyseal hormones: novel actors of striated muscle development and homeostasis
}

\author{
Alessandra Costa (1,2), Eleonora Rossi (1), Bianca Maria Scicchitano (1,2,3), Dario Coletti \\ $(1,2)$, Viviana Moresi (1), Sergio Adamo $(1,2)$ \\ (1) Histology and Medical Embryology Section, Dept. AHFMO, Sapienza University, Rome, \\ Italy; (2) I.I.M., Interuniversity Institute of Myology; (3) Institute of Histology and \\ Embryology, Catholic University School of Medicine, Rome, Italy
}

\begin{abstract}
Since the 1980's, novel functional roles of the neurohypophyseal hormones vasopressin and oxytocin have emerged. Several studies have investigated the effects of these two neurohormones on striated muscle tissues, both in vitro and in vivo. The effects of vasopressin on skeletal myogenic cells, developing muscle and muscle homeostasis have been documented. Oxytocin appears to have a greater influence on cardiomyocite differentiation and heart homeostasis. This review summarizes the studies on these novel roles of the two neurohypophyseal hormones, and open the possibility of new therapeutic approaches for diseases affecting striated muscle.

Key Words: Arginine-vasopressin, oxytocin, skeletal myogenic cells and cardiomyocite differentiation and homeostasis, receptors and signal transduction mechanisms
\end{abstract}

Eur J Trans Myol - Basic Appl Myol 2014; 24 (3): 217-225

Arginine-vasopressin (AVP), also known as antidiuretic hormone, and oxytocin (OT) are two neurohormones released by the posterior hypophysis.

These nonapeptides are synthesized in magnocellular neurons of the paraventricular and supraoptic nuclei of the hypothalamus. ${ }^{1}$ They are processed along the axonal projections to the neurohypophysis, stored in secretory vesicles and released, in response to a plethora of physiological stimuli, into the bloodstream, through which they reach distant targets and participate in the regulation of numerous functions that are crucial for survival. ${ }^{2,3}$ The only difference between AVP and OT lies in two amino acids. However, despite their structural similarity, these two neurohormones exert different physiological functions.

In mammals, OT plays an important role in reproduction by inducing uterus contraction upon labor and by increasing milk delivery during lactation. ${ }^{4,5}$ OT is also released in the brain, where it acts as a neuromodulator. Animal and human studies demonstrate that OT plays a role in social and sexual behaviors, in mood regulation in humans (likely through inhibition of amygdala activity), as well as in some behavioral disturbances, such as autism and obsessive-compulsive disorders. ${ }^{6-10}$ AVP, on the other hand, plays an essential role in regulating whole-body water balance, predominantly by modulating the expression and function of the water channel aquaporin-2 and of the urea transporters in the epithelial cells of the nephron collecting duct. ${ }^{11}$ In addition, AVP is a vasoconstrictor that increases peripheral vascular resistance, leading to increased arterial blood pressure. ${ }^{3}$ Like OT, AVP exerts direct effects on the brain: experiments on cognitive processes have demonstrated that both neurohormones enhance memory and attention. ${ }^{6,8}$ Unlike OT, AVP exerts an excitatory action on neural transmission. ${ }^{2}$

In addition to their well-documented role in the CNS, AVP and OT play important biological roles in many peripheral organs and tissues. While AVP mediates stress by triggering adrenocorticotropic hormone $(\mathrm{ACTH})$ release, OT exhibits anti-stress properties. Moreover, both AVP and OT play a marked role in cardiac homeostasis. AVP has been found to make a significant contribution to the progression of hypertension and heart failure, while OT has been found to reduce blood pressure and promote cardiac healing. ${ }^{3,12,13}$ The actions of OT include water body balance, modulation of the parasympathetic system, induction of NO-dependent vasodilatation, endothelial cell growth and anti-inflammatory response.

In addition, the extensive presence of a functional OT system in the early developing heart and the capacity of OT to induce the differentiation of mouse embryonic stem cells into cardiomyocytes provide 
additional evidence of its role in the cardiovascular system. $^{14}$

Until a few decades ago, neurohypophyseal hormones were not known to exert effects on skeletal muscle. This topic has emerged as a novel subject in the last three decades following studies conducted in various laboratories, including ours. In 1982, Wakelam et al. documented a modest effect of AVP on carbohydrate metabolism in chick embryo muscle cell cultures, ${ }^{15}$ and later demonstrated the presence of functional AVP receptors in the rat myogenic L6 cell line. ${ }^{16}$ AVP levels were subsequently reported to be very high in developing skeletal muscle, and to decline during gestation, reaching very low levels at birth, ${ }^{17}$ thus suggesting that this hormone is involved in the early phases of muscle development. More recently, Breton et al. provided evidence that functional OT receptors are present in human primary myoblasts. ${ }^{18}$

These observations suggest that neurohypophyseal hormones may play a role during skeletal myogenesis and might help maintain skeletal muscle homeostasis. In our first paper on this subject, ${ }^{19}$ we reported important responses elicited by AVP in myogenic cells, which included generation of inositol phosphates and increased cytosolic free calcium concentrations. We also demonstrated that the responsiveness to AVP of different myogenic cell lines and clones positively correlated with their myogenic potential.

In this review, we summarize the effects that AVP and OT have been reported to exert on skeletal muscle in recent decades to highlight the role of these hormones in skeletal muscle biology.

\section{Neurohypophyseal hormone receptors}

Both oxytocin and vasopressin receptors are heptahelical transmembrane proteins that activate $\mathrm{G}$ proteins. Three types of AVP receptor are known: V1a, $\mathrm{V} 1 \mathrm{~b}$ and $\mathrm{V} 2$, which differ in localization, function and signal transduction mechanisms. The V1a and V1b receptors activate phospholipases $\mathrm{A} 2, \mathrm{C}$ and $\mathrm{D} ; \mathrm{V} 2$ receptors regulate adenylate cyclase activity. ${ }^{20,21}$ The $\mathrm{V} 1 \mathrm{a}$ receptors $(\mathrm{V} 1 \mathrm{aR})$ are located on both vascular smooth muscle cells and cardiomyocytes, and have been shown to modulate blood vessel vasoconstriction and myocardial function. ${ }^{22,23}$ These receptors are also expressed in many other organs or cells, such as liver, platelets, lymphocytes and monocytes, the central nervous system, reproductive organs, retinal epithelium, renal mesangial cells, as well as in many cell lines, including L6 rat myoblasts. Hiroyama et al. generated a knock-out mouse for $\mathrm{V} 1 \mathrm{aR}\left(\mathrm{V}_{\mathrm{anR}}^{-/-}\right)$that exhibits an impairment in spatial memory, low blood pressure, hypermetabolism of fat and insulin resistance. ${ }^{13}$ Interestingly, $\mathrm{V} 1 \mathrm{aR}^{-/-}$mice also displayed hyperammonaemia, which was due to increased protein catabolism, thereby suggesting that AVP plays a role in regulating the balance between protein synthesis and degradation. ${ }^{13}$
The V1b subtype was recently identified in the anterior pituitary, where it stimulates the release of ACTH. The V2 receptor is mainly expressed in the kidney and is responsible for the antidiuretic function of the hormone. ${ }^{20}$ AVP binding promotes the phosphorylation and desensitization of $\mathrm{V} 1 \mathrm{a}$ and $\mathrm{V} 2$ receptors. The $\mathrm{V} 1 \mathrm{aR}$ is internalized in the cytosol, dephosphorylated and rapidly returns to the cellular surface. Conversely, V2 receptors associate with $\beta$-arrestin and remain in the cytosol for a longer period of time. ${ }^{20,24}$ The OT receptor (OTR), similarly to V2 receptors, is internalized and is mainly associated with the clathrindependent pathway. The expression of OTR has been detected and characterized in brain, myometrium, mammary gland, endometrium, decidua, ovary, as well as in the male reproductive apparatus, thymus, heart and kidney, ${ }^{25}$ on the surface of vascular endothelial cells, osteoblasts, cardiomyocytes and human satellite cells. $^{18}$ Owing to their homology in sequence and structure, both AVP and OT receptors bind AVP and OT with different affinities: AVP binds to OTR in a non-selective manner, whereas OT interacts with V1aR with a low efficiency. ${ }^{20}$

\section{Biological effects of AVP and OT on myogenic cells in culture}

The first evidence of the biological effects of neurohypophyseal hormones as modulators of skeletal muscle differentiation came from cell culture experiments. In 1995, Nervi et al. showed that the addition of AVP to the culture medium of L6 or L5 myoblasts or mouse satellite cells resulted in a marked increase in the percentage of fusion and in the formation of hypertrophic myotubes, in the absence of significant effects on cell proliferation. The expression of early (Myf-5 and myogenin) and late (myosin, acetylcholine receptor [AchR] subunits) myogenic differentiation markers was increased by the neurohypophyseal hormone in a structure- and concentration-dependent fashion. ${ }^{26}$ Using a serum-free culture medium for L6 and L5 myoblasts and for mouse satellite cells, we demonstrated that AVP is sufficient to induce robust myogenic differentiation. ${ }^{27}$ These data confirmed the role of AVP as a positive regulator of terminal differentiation in myogenic cells. Recent in vitro studies have demonstrated that the expression of the $\mathrm{V} 1 \mathrm{aR}$ receptor is modulated during myogenic differentiation, ${ }^{28}$ which points to the physiologic involvement of AVP in myogenesis. This hypothesis is supported by data highlighting the presence of high levels of immunoreactive AVP in both human and mouse embryonic and fetal muscles, ${ }^{17}$ as well as by the presence of a vasopressin-like peptide in the mammalian sympathetic nervous system. ${ }^{29}$ Interestingly, as reported by Nervi, it is not only AVP, but also OT that is involved in myogenic differentiation. ${ }^{26}$ Indeed, evidence has been provided of the presence of functional OTRs in human myoblast 


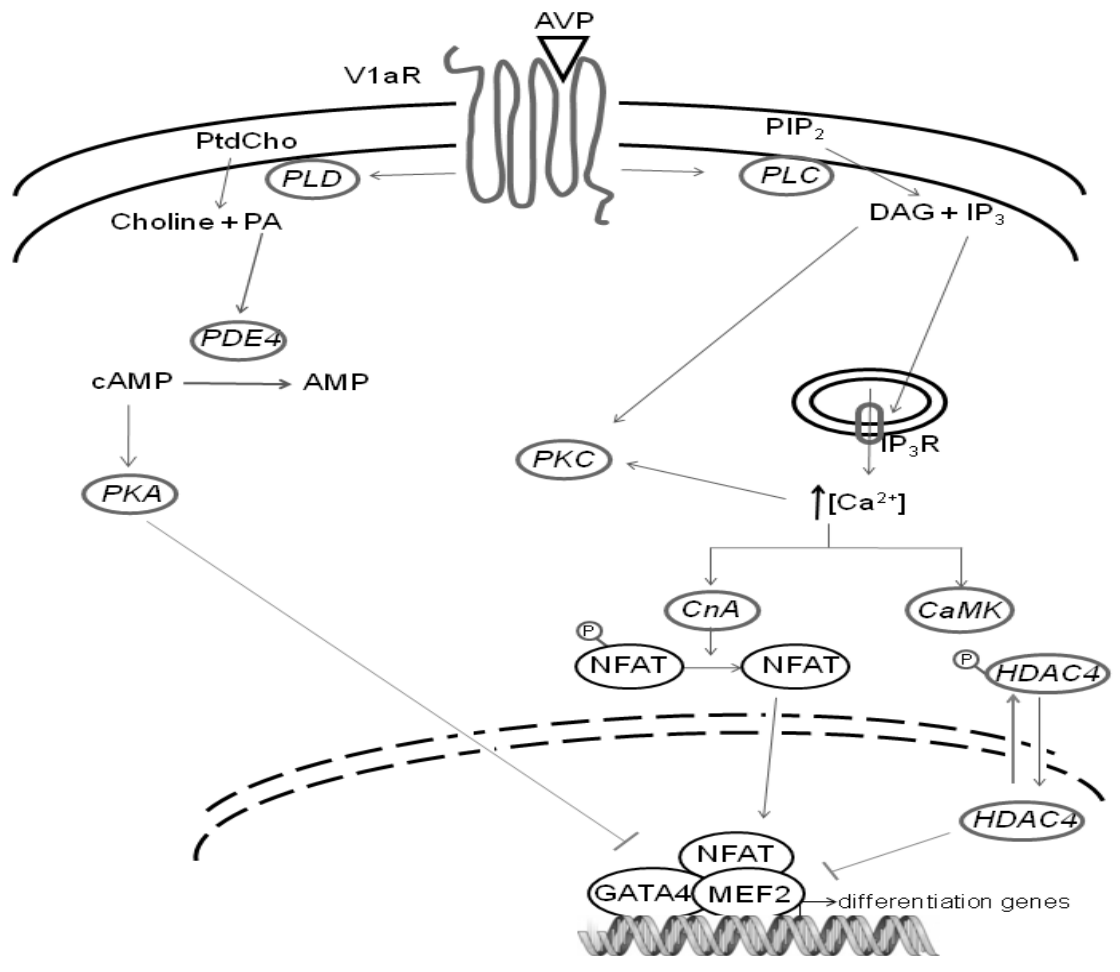

Fig 1. Schematic representation of AVP-dependent signaling pathways in myogenic cells. The transduction of AVP signal in myogenic cells involves the activation of PLC and PLD. PLC hydrolyzes phosphatidylinositol 4,5bisphosphate, producing the second messengers DAG and IP3. DAG also derives from PA produced by PtdCho via PLD. DAG activates the protein kinase C (PKC), while IP3 is responsible for $\mathrm{Ca}^{2+}$ release from the endoplasmic reticulum into the cytosol. An increased intracellular cytosolic $\mathrm{Ca}^{2+}$ concentration activates the CaMK pathway, thus inducing cytosolic compartmentalization of class II HDACs. Calcium signaling also activates CnA, which in turn promotes the dephosphorylation and the consequent nuclear import of the NFAT family. In the nucleus, NFAT gives rise to the formation of multifactorial complexes with MEF2 and GATA2 in the promoter region of muscle specific genes. The absence of class II HDACs is also likely to promote this transcriptional activation, leading to the activation of the skeletal muscle differentiation program. AVP also interferes with the cAMP signaling system: PA (produced by PLD-dependent hydrolysis of PtdCho) activates PDE4, which in turn promotes CAMP breakdown, thus inhibiting cAMP-dependent activation of PKA, a kinase known to negatively regulate myogenic differentiation.

obtained from clonal cultures of postnatal satellite cells. $^{18}$

\section{Biological effects of neurohypophyseal hormones on cardiomyocytes}

A new role for AVP in cardiomyogenesis has emerged in recent years. Several studies have shown that AVP increases the rate of protein synthesis in cardiomyocytes of mice and rats, thereby exerting a hypertrophic effect mediated by V1aR. ${ }^{13,30}$ The effect of AVP on differentiation has been reported on atrial and ventricular precursor cells through the AVP receptors V2R and V1aR, respectively. ${ }^{31}$ Taken together, these findings highlight the possible role of this nonapeptide in cardiomyogenesis and heart homeostasis.

Evidence of the role of OT even in cardiac differentiation has emerged from experiments involving stem cell lines. Paquinet al. examined the role of OT using P19 embryonic stem cells which, instead of giving rise to all germ layer cell derivatives, differentiated into beating cardiomyocytes after treatment with $0.1 \mu \mathrm{M} \mathrm{OT} .{ }^{32}$ Furthermore, it was demonstrated that the cardiogenic action of OT is specifically mediated by OTR. In addition, Hatami et al. confirmed that OT treatment of embryonic stem cells induces them to differentiate into functional cardiomyocytes that are responsive to cardioactive 
drugs, with the typical ultrastructure and expression of multiple cardiac-associated molecular markers. ${ }^{33}$

Collectively, the results of these studies, most of which were performed in culture, strongly suggest that both AVP and OT are involved in the processes of myogenic and cardiomyogenic differentiation.

\section{Neurohypophyseal hormones activate a complex} network of intracellular signals in myogenic cells

The discovery of the expression of OT and AVP receptors in many myogenic cells, together with the knowledge of the decrease in AVP concentrations during gestation ${ }^{17}$ and their increase upon physical exercise (see section: Physiological role of neurohypophyseal hormones on muscle development and homeostasis), point to a physiological role of OT and AVP in skeletal muscle development or homeostasis. This has, in turn, led to studies being conducted to shed light on signaling in this tissue. Most of these studies have focused on AVP-dependent signaling, which is now well characterized. Conversely, little is known about OT signaling in skeletal muscle, the only exception being that OT regulates glucose uptake via AMPK in a calciumdependant manner. ${ }^{34}$

Role of phospholipases $C$ and $D$ in vasopressindependent myogenic differentiation

Transduction of the AVP signal in myogenic cells (Figure 1) involves the activation of phospholipases $\mathrm{C}$ and D (PLC, PLD). AVP induces concentrationdependent (in the $0.1 \mathrm{nM}-1 \mu \mathrm{M}$ range) stimulation of PLC activity and regulates the intracellular $\mathrm{pH}$ with mechanisms involving both $\mathrm{Na}^{+}$and anion transports across the plasma membrane. Inositol 1,4,5trisphosphate (IP3) production is maximally stimulated within 2-5 sec of AVP treatment, immediately followed by $\mathrm{Ca}^{2+}$ release from intracellular stores. Activation of protein kinase $\mathrm{C}$ by phorbol esters, as well as the administration of antagonists of AVP for the binding of V1Rs, inhibits these responses. ${ }^{19}$

AVP stimulation of myogenic cells also results in the activation of PLD-dependent phosphatidylcholine (PtdCho) breakdown. AVP induces the monophasic generation of phosphatidic acid (PA) and the biphasic increase in sn-1,2-diacylglycerol (DAG), consisting in a rapid peak (within $5 \mathrm{sec}$ of AVP treatment, resulting from PLC activity), followed by a sustained phase. PLD activation is elicited at AVP concentrations that are two orders of magnitude lower than those required for PLC activation $(E C 50=0.4 \mathrm{nM}$ vs $\mathrm{EC} 50=50 \mathrm{nM}$, respectively), ${ }^{35}$ thereby suggesting that this pathway may play a role at physiological concentrations of the hormone.

AVP-dependent activation of PLD causes hydrolysis of conspicuous amounts of plasma membrane PtdCho in L6 myoblasts. PtdCho de novo synthesis is rapidly activated, along with microtubule dependent vesicle traffic. By fusing newly formed, PtdCho enriched vesicles with the plasma membrane, the physiological level of PtdCho is rapidly (within 5 min) restored. ${ }^{36,37}$

cAMP hydrolysis is required for vasopressindependent stimulation of myogenesis

AVP treatment of myogenic cells not only induces an obvious cross-talk between PLD and PLC signaling pathways, but also interferes with the cAMP signaling system. It is well known that [cAMP-dependent protein kinase (PKA)] negatively regulates myogenic differentiation by inhibiting the activity of myogenic helix-loop-helix transcription factors 38,39 In AVP treated L6 cells, selective stimulation of specific cAMP-phosphodiesterase isoforms, such as phosphodiesterase E4 (PDE4), has been observed in response to PA production by PLD. ${ }^{40}$ Accordingly, cAMP levels and PKA activity are down-regulated by AVP, ultimately allowing the nuclear translocation and transcriptional activity of myogenesis regulatory factors, myogenin and MyoD. ${ }^{41,42}$ Interestingly, in the L6 myogenic cell line, which is maintained quiescent by serum deprivation, the addition of AVP restores the ability of IGFI to induce differentiation through modulation of PDE4 activity. ${ }^{43}$ Furthermore, unlike IGFs, AVP stimulates myoblast fusion and does not increase proliferation. ${ }^{26}$ AVP is reported to exert its pro-myogenic effects not only on L6 myoblasts but also on primary satellite cells, by up-regulating the expression of muscle-specific genes, such as Myf-5, myogenin, myosin heavy chain (MHC), muscle creatine kinase (MCK) and AChRs. ${ }^{43,44}$

Vasopressin-dependent myogenic cell differentiation is mediated by both $\mathrm{Ca}^{2+} /$ calmodulin-dependent kinase and calcineurin pathways.

Many transcription factors play crucial roles during myogenesis. In addition to Myf-5, the MEF2 family transcription factors recognizes specific DNA elements on the promoter of muscle genes. Skeletal muscle cells treated with $0.1 \mu \mathrm{M}$ AVP displayed an induction of MEF2A and MEF2C, which occurred concomitantly with myogenin expression, during myoblast to myotube transition. ${ }^{44}$ The molecular mechanism of this effect involves the dissociation of the class II histone deacetylases (HDACs) from MEF2. Indeed, translocation of class II HDACs from the nucleus to the cytosol allows the transcriptional activation of MEF2. ${ }^{45}$ The AVP-dependent increase in intracellular cytosolic $\mathrm{Ca}^{2+}$ concentrations activates the calcium/calmodulin-dependent protein kinase (CaMK) pathway. This is sufficient to induce cytosolic compartmentalization of class II HDACs, as demonstrated by the reduced nuclear export of class II HDACs and consequent MEF2 activation induced by CaMK inhibitors. ${ }^{44}$ Moreover, during AVP-stimulated myogenesis, we noted an increase in the acetylation levels of histone $\mathrm{H} 3$ associated with the myogenin 


\section{Neurohypophyseal hormones in muscle development and homeostasis}

Eur J Trans Myol - Basic Appl Myol 2014; 24 (3): 217-225

promoter and the MCK enhancer. However, the AVPdependent full activation of the myogenic program cannot be sustained by CaMK alone. ${ }^{46}$ An additional calcium-dependent pathway involves calcineurin (CnA), a serine/threonine phosphatase responsible for the dephosphorylation and the consequent nuclear import of Nuclear Factor of Activated T-cells (NFAT) family. ${ }^{47}$ Myoblasts treated with cyclosporine A (CsA), a partial inhibitor of $\mathrm{CnA}$, or transiently transfected with the dominant negative construct $\mathrm{CnA}-\mathrm{KO}$ displayed a dramatic down-regulation of myogenic differentiation at both the morphological and the molecular levels. Moreover, CsA treatment of L6 cells inhibited the expression of MEF2, NFATc1 and GATA2 (a muscle isoform of the zinc-finger GATA family); conversely, AVP administration promoted accumulation of these three transcription factors in nuclei. ${ }^{46}$ Therefore, on the one hand, AVP stimulates the formation of multifactorial complexes, which involve MEF2, NFATc1 and GATA2, in the promoter or enhancer regions of muscle specific genes via the calcineurin pathway; on the other hand, the AVPdependent activation of CaMK promotes histone acetylation in the same DNA regions, leading to full expression of the myogenic differentiation program.

\section{Physiological role of neurohypophyseal hormones on muscle development and homeostasis}

Muscle function is highly dependent on and coordinated with blood flow and pressure. Indeed, muscle activity induces an exercise pressor reflex, a regulatory loop that AVP helps to regulate at the neuro-endocrine level. ${ }^{48,49}$ It is worth noting that skeletal muscle contraction (e.g. induced by exercise) or lack of contraction (e.g., following denervation) can influence AVP plasma levels. One of the latest examples of this effect is found in the study by Liviakis et al., which demonstrates that short-term static muscle contraction increases plasma AVP. ${ }^{50}$ In that study, higher levels of contraction $(>70 \%$ of maximal tension production) increased plasma AVP by approximately $100 \%$, whereas low intensity contraction failed to alter systemic concentrations of this peptide. The reflex nature of this response was confirmed by the fact that high-intensity contractioninduced increases in plasma levels of AVP were not associated with changes in other important variables that stimulate the release of this peptide (e.g. increases in osmolality and body temperature and decreases in plasma volume), and that these increases were eliminated by sensory denervation of the contracting muscle. ${ }^{50}$ Interestingly, several other reports indicate that resistance muscular exercise, which represents a hypertrophic signal, results in a significant increase in circulating AVP, in both in human and other mammals, thus posing the theoretical basis for the physiological regulation of muscle hypertrophy by neurohypophyseal hormones. ${ }^{46,51-54}$ Furthermore, the calcineurin pathway, which is strongly stimulated by AVP, has been shown to be essential for muscle regeneration in normal and dystrophic animals. ${ }^{55}$ Lastly, determination of muscle specificity, which is required in muscle development, is finely regulated by the smooth muscle-specific 22$\mathrm{kDa}$ protein, which is in turn regulated by AVP. ${ }^{56,57} \mathrm{~A}$ large body of evidence shows that the AVP system is impaired in several neuromuscular diseases, such as amyotrophic lateral sclerosis and multiple sclerosis. ${ }^{58,59}$ These data strengthen the hypothesis that AVP plays a physiological role in skeletal muscle homeostasis.

Recent findings from our laboratory demonstrate that muscle-specific stimulation of AVP signaling induces acceleration of satellite cell activation and earlier stimulation of the specific pathway involved in muscle regeneration. V1aR over-expression in murine injured skeletal muscle is responsible for an anticipation and amplification of the regeneration program. ${ }^{60,61}$ The rapid recovery of injured skeletal muscle is associated with the rapid resolution of inflammation, the earlier activation and fusion of satellite cells, and the faster formation of regenerating fibers. Indeed, in V1aR overexpressing muscle, the expression levels of regeneration/differentiation markers such as Pax7, embryonic MHC and myogenin, as well as the expression and activity of calcineurin and its downstream targets IL-4, are strongly up-regulated and hastened when compared with mock transfected muscle. ${ }^{60}$ In addition, we showed that intramuscular AVP injection promotes skeletal muscle regeneration following focal injury and rescues muscle repair and force, even when muscle recovery is hampered by concomitant Tumor Necrosis Factor (TNF)] treatment. ${ }^{62}$ In particular, we demonstrated cross-talk at the Heat shock protein 70 (Hsp70) level in muscle: while AVP (and pro-myogenic cues) upregulate Hsp70 levels, TNF (and pro-inflammatory cues) downregulate them. Hsp70 interacts with the stem cell marker $\mathrm{PW} 1 / \mathrm{Peg} 3$, which is, in turn, responsible for TNFmediated, caspase-dependent inhibition of stem cell differentiation in regenerating muscle, ${ }^{63}$ thus suggesting that AVP rescues stem cell participation in muscle regeneration via Hsp70-mediated anti-caspase activity. ${ }^{62}$ On the basis of these results, we may conclude that AVP-V1aR signaling plays an important role in skeletal muscle development and homeostasis.

Several recent studies have pointed to a physiological role of neurohypophyseal hormones in muscle homeostasis by providing evidence of the role of OT in muscle growth. As shown by De Jager et al., treatment of cattle skeletal muscle with anabolic steroids unexpectedly led to a high expression of the OTencoding mRNA, accompanied by a high level of circulating OT. ${ }^{64}$ The authors hypothesized that OT might be involved in mediating the anabolic effects of steroids, considering it to be responsible for the increased muscle mass. Although sex steroid status is known to be related to OT, ${ }^{25}$ and skeletal muscle is 
known to express functional OTRs, ${ }^{18}$ the endogenous production of the hormone by skeletal muscle was unexpected. ${ }^{64}$ Up-regulation of OT mRNA expression was also detected in wethers treated with steroid hormones. $^{65}$

\section{Physiological role of neurohypophyseal hormones on cardiac muscle development and homeostasis}

Many reports have also highlighted the effects of AVP on cardiac muscle homeostasis. High levels of immunoreactive AVP are present in newborn rat heart and decline in adult rat heart, which suggests that the AVP system plays a role in early rat maturation. ${ }^{66}$ Hiroyama et al. reported that the AVP-V1aR signaling pathways play a significant role in cardiac hypertrophy in response to pressure overload, a finding that is consistent with the results of studies on the hypertrophic effect of AVP on cultured neonatal heart cells from mice and rats. ${ }^{13,30}$ Hupf et al. also detected AVP synthesis in pressure-overloaded, isolated and perfused rat hearts. ${ }^{67}$ Other studies have reported that the heart raises AVP plasma levels in cardiac failure, ${ }^{12,68,69}$ hypothesizing the use of AVP antagonist in the treatment of this pathology. ${ }^{70-72}$ In recent decades, it has been established that OT plays a significant role in cardiac muscle homeostasis. OTRs are present in rat atria and ventricle and mediate the release of andropin, exerting a negative chronotropic effect. ${ }^{73}$ The heart is also capable of synthesizing and releasing structurally identical OT from the same gene as the neurohypophyseal OT. The presence of OT and OTRs in all the chambers suggests that this peptide plays an autocrine and/or paracrine role in heart development and homeostasis. ${ }^{74}$

\section{Conclusions}

A large body of evidence that has emerged in the last three decades indicates that neurohypophyseal hormones, i.e. AVP and OT, are involved in muscle development and homeostasis. The effects of AVP on myogenic cells, primary stem cells and whole skeletal muscle have been widely investigated. Less is known about the signaling and function of OT in this tissue. Taken together, these observations suggest that it may be possible to exploit AVP to treat skeletal muscle disease conditions (such as dystrophies, cachexia and atrophy). OT instead appears to have a greater effect on cardiomyocyte differentiation and heart homeostasis, which suggests it may be a good candidate in the treatment of various cardiac diseases. The findings reported in this review, which summarizes novel pathways that regulate muscle differentiation and homeostasis, radically change the canonical roles of neurohypophyseal hormones and pave the way for a more thorough understanding of the functions of AVP and OT.

\section{Acknowledgements}

Some of the authors would not be the same people, either scientifically or personally, had they not met Prof. Ugo Carraro at a given moment in their careers. We are indeed deeply indebted to him for being our long-lasting, non-denervated mentor. Some of us, who have been lucky enough to enjoy the company of our colleague Ugo, whose hallmark characteristics are sagacious feedback and iconoclastic outbursts, look forward to sharing new experiences and exciting discussions for many years to come.

We are also extremely grateful to Dr. Paola Aprile, a member of our lab, for her contribution. The authors are supported by the following agencies and grants: MIUR PRIN, Sapienza University of Rome (to SA); AFM, ANR, NIH and UPMC Emergence (to DC).

\section{Conflicts of Interest}

The authors declare that there is no conflict of interests regarding the publication of this article.

\section{Corresponding Author}

Sergio Adamo, Sez. Istologia ed Embriologia Medica Via A. Scarpa 00161 Rome, Italy

E-mail: sergio.adamo@uniroma1.it

\section{References}

1. Landgraf R, Neumann ID. Vasopressin and oxytocin release within the brain: a dynamic concept of multiple and variable modes of neuropeptide communication. Front Neuroendocrinol 2004;25:150-76.

2. Meyer-Lindenberg A, Domes G, Kirsch P, Heinrichs M. Oxytocin and vasopressin in the human brain: social neuropeptides for translational medicine. Nat Rev Neurosci 2011; 12:524-38.

3. Japundžić-Žigon N. Vasopressin and oxytocin in control of the cardiovascular system. Curr Neuropharmacol 2013;11:218-30.

4. Mitchell BF, Schmid B. Oxytocin and its receptor in the process of parturition. J Soc Gynecol Investig 2001;8:122-33.

5. Bealer SL, Armstrong WE, Crowley WR. Oxytocin release in magnocellular nuclei: neurochemical mediators and functional significance during gestation. Am J Physiol Regul Integr Comp Physiol 2010;299:R452-8.

6. Neumann ID, Landgraf R, Veenema AH. Central vasopressin and oxytocin release: regulation of complex social behaviours. Prog Brain Res 2008;170:261-276.

7. Lukas M, Neumann ID. Oxytocin and vasopressin in rodent behaviors related to social dysfunctions in autism spectrum disorders. Behav Brain Res 2013;251:85-94. 


\section{Neurohypophyseal hormones in muscle development and homeostasis}

Eur J Trans Myol - Basic Appl Myol 2014; 24 (3): 217-225

8. Carter CS. Oxytocin pathways and the evolution of human behavior. Annu Rev Psychol 2014;65:17-39.

9. Viero C, Shibuya I, Kitamura N, et al. REVIEW: Oxytocin: Crossing the bridge between basic science and pharmacotherapy. CNS Neurosci Ther 2010;16:e138-56.

10. Insel TR. The challenge of translation in social neuroscience: a review of oxytocin, vasopressin, and affiliative behavior. Neuron 2010;65:768-79.

11. Moeller HB, Fenton RA. Cell biology of vasopressin-regulated aquaporin-2 trafficking. Pflugers Arch 2012:464:133-44.

12. Lee C.R, Watkins ML, Patterson JH, et al. Vasopressin: a new target for the treatment of heart failure. Am Heart J 2003;146:9-18.

13. Hiroyama $\mathrm{M}$, Wang $\mathrm{S}$, Aoyagi $\mathrm{T}$, et al. Vasopressin promotes cardiomyocyte hypertrophy via the vasopressin V1A receptor in neonatal mice. Eur J Pharmacol 2007;559:89-97.

14. Gutkowska J, Jankowski M. Oxytocin revisited: its role in cardiovascular regulation. $\mathbf{J}$ Neuroendocrinol 2012;24:599-608.

15. Wakelam MJ, Pette D. The control of glucose 1,6-bisphosphate by developmental state and hormonal stimulation in cultured muscle tissue. Biochem J 1982;204:765-9.

16. Wakelam MJ, Patterson S, and M. R. Hanley MR. L6 skeletal muscle cells have functional V1vasopressin receptors coupled to stimulated inositol phospholipid metabolism. FEBS Lett 1987;210:181-4.

17. Smith A, Stephen RI, Arkley MM, McIntosh N. Immunoreactive arginine vasopressin in human fetal and neonatal skeletal muscle. Early Hum Dev 1992;28:215-222.

18. Breton C, Haenggeli C, Barberis $\mathrm{C}$, et al. Presence of functional oxytocin receptors in cultured human myoblasts. J Clin Endocrinol Metab 2002;87:1415-8.

19. Teti A, Naro F, Molinaro M, Adamo S. Transduction of arginine vasopressin signal in skeletal myogenic cells. Am J Physiol 1993;265:C113-21.

20. Koshimizu T, Nakamura K, Egashira N, et al. Vasopressin V1a and V1b receptors: from molecules to physiological systems. Physiol Rev 2012;92:1813-64.

21. Thibonnier M, Graves MK, Wagner MS, et al. Structure, sequence, expression, and chromosomal localization of the human V1a vasopressin receptor gene. Genomics 1996;31: 327-34.

22. Penit J, Faure M, Jard S. Vasopressin and angiotensin II receptors in rat aortic smooth muscle cells in culture. Am J Physiol 1983;244, E72-82.
23. Aoyagi T, Koshimizu T, Tanoue A. Vasopressin regulation of blood pressure and volume: findings from V1a receptor-deficient mice. Kidney Int 2009;76:1035-9.

24. Birnbaumer M. Vasopressin receptors. Trends Endocrinol Metab 2000;11:406-10.

25. Gimpl G, Fahrenholz F. The oxytocin receptor system: structure, function, and regulation. Physiol Rev 2001;81:629-83.

26. Nervi C, Benedetti L, Minasi A, et al. Argininevasopressin induces differentiation of skeletal myogenic cells and up-regulation of myogenin and Myf-5. Cell Growth Differ 1995. 6: 81-89.

27. Minotti S, Scicchitano BM, Nervi C, et al. Vasopressin and insulin-like growth factors synergistically induce myogenesis in serum-free medium. Cell Growth Differ 1998;9:155-63.

28. Alvisi M, De Arcangelis V, Ciccone L, et al. V1a vasopressin receptor expression is modulated during myogenic differentiation. Differentiation 2008;76:371-80.

29. Hanley MR, Benton HP, Lightman SL, et al. A vasopressin-like peptide in the mammalian sympathetic nervous system. Nature 1984;309: 258-261.

30. Nakamura Y, Haneda T, Osaki J, et al. "Hypertrophic growth of cultured neonatal rat heart cells mediated by vasopressin V(1A) receptor. Eur J Pharmacol 2000;391:39-48.

31. Gassanov N, Jankowski M, Danalache B, et al. Arginine vasopressin-mediated cardiac differentiation: insights into the role of its receptors and nitric oxide signaling. J Biol Chem 2007;282:11255-11265.

32. Paquin J, Danalache BA, Jankowski M, et al. Oxytocin induces differentiation of P19 embryonic stem cells to cardiomyocytes Proc Natl Acad Sci U S A 2002;99:9550-5.

33. Hatami L, Valojerdi MR, Mowla SJ. Effects of oxytocin on cardiomyocyte differentiation from mouse embryonic stem cells. Int $\mathbf{J}$ Cardiol 2007;117;80-89.

34 Lee ES, Uhm K-O, Lee YM, et al. Oxytocin stimulates glucose uptake in skeletal muscle cells through the calcium-CaMKK-AMPK pathway. Regul Pept2008;71-4.

35. Naro F, Donchenko V, Minotti S. et al. Role of phospholipase $\mathrm{C}$ and $\mathrm{D}$ signaling pathways in vasopressin-dependent myogenic differentiation. J Cell Physiol 1997; 171:34-42.

36. Coletti D, Palleschi S, Silvestroni L, et al. Surface remodeling associated with vasopressin-induced membrane traffic in L6 myogenic cells. Arch Histol Cytol 2000;63:441-9.

37. Coletti D, Silvestroni L, Naro F, et al. Vesiclemediated phosphatidylcholine reapposition to the plasma membrane following hormone-induced 


\section{Neurohypophyseal hormones in muscle development and homeostasis}

Eur J Trans Myol - Basic Appl Myol 2014; 24 (3): 217-225

phospholipase D activation. Exp Cell Res 2000;256:94-104.

38. Li L, Heller-Harrison R, Czech M, Olson EN. Cyclic AMP-dependent protein kinase inhibits the activity of myogenic helix-loop-helix proteins. Mol Cell Biol 1992;12:4478-4485.

39. Winter B, Braun T, Arnold HH. cAMP-dependent protein kinase represses myogenic differentiation and the activity of the muscle-specific helix-loophelix transcription factors Myf-5 and MyoD. J Biol Chem 1993;268:9869-9878.

40. Némoz G, Sette C, Conti M. Selective activation of rolipram-sensitive, cAMP-specific phosphordiesterase isoforms by phosphatidic acid. Mol Pharmacol 1997;51:242-9.

41. Naro F, De Arcangelis V, Sette C, et al. A bimodal modulation of the cAMP pathway is involved in the control of myogenic differentiation in 16 cells. $J$ Biol Chem 2003;278:49308-15.

42. Naro F, Sette C, Vicini E, et al. Involvement of type 4 cAMP-phosphodiesterase in the myogenic differentiation of L6 cells. Mol Biol Cell 1999;10:4355-67.

43. De Arcangelis V, Coletti D, Conti M, et al. IGF-Iinduced differentiation of L6 myogenic cells requires the activity of cAMP-phosphodiesterase. Mol Biol Cell 2003;14:1392-404.

44. Scicchitano BM, Spath L, Musaro A, et al. AVP induces myogenesis through the transcriptional activation of the myocyte enhancer factor 2," Mol. Endocrinol 2002;16:1407-1416.

45. McKinsey TA, Zhang CL, Olson EN. Activation of the myocyte enhancer factor-2 transcription factor by calcium/calmodulin-dependent protein kinase-stimulated binding of 14-3-3 to histone deacetylase 5. Proc Natl Acad Sci USA 2000;97:14400-5.

46. Scicchitano BM, Spath L, Musarò A, et al. Vasopressin-dependent myogenic cell differentiation is mediated by both $\mathrm{Ca} 2+/$ calmodulin-dependent kinase and calcineurin pathways. Mol Biol Cell 2005; $16: 3632-41$.

47. Batiuk TD, Halloran OF. The downstream consequences of calcineurin inhibition. Transplant Proc 2002;29:1239-40.

48. Stebbins CL, Bonigut S, Liviakis LR, Munch PA. Vasopressin acts in the area postrema to attenuate the exercise pressor reflex in anesthetized cats. Am J Physiol 1998;274:H2116-22.

49. Mitchell JH, Kaufman MP, Iwamoto GA. The exercise pressor reflex: its cardiovascular effects, afferent mechanisms, and central pathways. Annu Rev Physiol 1983;45:229-42.

50. Liviakis LR, Stebbins CL. Static contraction causes a reflex-induced release of arginine vasopressin in anesthetized cats. Brain Res. Bull 2000;53:233-238

51. Melin P, Lundin S, Vilhardt H, et al. Antidiuretic agonism and antagonism of some O-alkylated analogues of vasopressin containing 2-Oalkylated tyrosine. Eur J Pharmacol 1980;67:173177.

52. Alexander SL, Irvine CH, Ellis MJ, Donald RA. The effect of acute exercise on the secretion of corticotropin-releasing factor, arginine vasopressin, and adrenocorticotropin as measured in pituitary venous blood from the horse. Endocrinology 1991;128:65-72.

53. Melin B, Jimenez C, Savourey G, et al. Effects of hydration state on hormonal and renal responses during moderate exercise in the heat Eur J Appl Physiol Occup Physiol 1997;76:320-327.

54. Convertino VA, Keil LC, Bernauer EM, Greenleaf JE. Plasma volume, osmolality, vasopressin, and renin activity during graded exercise in man. J Appl Physiol 1981;50:123-8.

55. Stupka N, Gregorevic P, Plant DR, Lynch GS. The calcineurin signal transduction pathway is essential for successful muscle regeneration in mdx dystrophic mice. Acta Neuropathol 2004;107: 299-310.

56. Chang PS, Li L, McAnally J, Olson EN. Muscle specificity encoded by specific serum response factor-binding sites. J Biol Chem 2001;276:17206-12.

57. Kaplan-Albuquerque N, Garat C, Van Putten V, Nemenoff RA. Regulation of SM22 alpha expression by arginine vasopressin and PDGF$\mathrm{BB}$ in vascular smooth muscle cells. Am J Physiol Heart Circ Physiol 2003;285:H1444-52.

58. Gonzalez de Aguilar JL, Gordon JW, Rene F, et al. A mouse model of familial amyotrophic lateral sclerosis expressing a mutant superoxide dismutase 1 shows evidence of disordered transport in the vasopressin hypothalamoneurohypophysial axis Eur J Neurosci 1999;11:4179-4187.

59. Michelson D, Stone L, Galliven E, et al. Multiple sclerosis is associated with alterations in hypothalamic-pituitary-adrenal axis function. J Clin Endocrinol Metab 1994;79:848-53.

60 Toschi A, Severi A, Coletti D, et al. Skeletal muscle regeneration in mice is stimulated by local overexpression of V1a-vasopressin receptor. Mol Endocrinol 2011;25:1661-73.

61. Toschi A, Murfuni I, Coletti D, et al. V1a AVP receptor is required for neurohypophyseal hormone-dependent differen-tiation in $\mathrm{C} 2 \mathrm{C} 12$ cells. Basic Appl Myol 2009;19:229-236.

62. Moresi V, Garcia-Alvarez G, Pristerà A, et al. Modulation of caspase activity regulates skeletal muscle regeneration and function in response to 


\section{Neurohypophyseal hormones in muscle development and homeostasis}

Eur J Trans Myol - Basic Appl Myol 2014; 24 (3): 217-225

vasopressin and tumor necrosis factor. PLoS One, 2009;4:e5570.

63. Moresi V, Pristerà A, Scicchitano BM, et al. Tumor necrosis factor-alpha inhibition of skeletal muscle regeneration is mediated by a caspasedependent stem cell response. Stem Cells 2008;26: 997-1008.

64. De Jager N, Hudson NJ, Reverter A, et al. Chronic exposure to anabolic steroids induces the muscle expression of oxytocin and a more than fiftyfold increase in circulating oxytocin in cattle. Physiol Genomics 2011;43;467-78.

65. Kongsuwan K, Knox MR, Allingham PG, et al. The effect of combination treatment with trenbolone acetate and estradiol-17 $\beta$ on skeletal muscle expression and plasma concentrations of oxytocin in sheep. Domest Anim Endocrinol 2012;43: 67-73.

66. Gutkowska J, Miszkurka M, Danalache B, et al. Functional arginine vasopressin system in early heart maturation. Am J Physiol Heart Circ Physiol 2007;293:H2262-H2270.

67. Hupf H, Grimm D, Riegger GA, Schunkert HH, Evidence for a vasopressin system in the rat heart. Circ Res 1999;84:365-370.

68. Goldsmith SR, Francis GS, Cowley AW, et al. Increased plasma arginine vasopressin levels in patients with congestive heart failure. J Am Coll Cardiol 1983;1:1385-1390.

69. Parmley WW. Neuroendocrine changes in heart failure and their clinical relevance. Clin Cardiol 1995;18:440-5.

70. Tahara A, Tomura Y, Wada KI, et al. Pharmacological profile of YM087, a novel potent nonpeptide vasopressin V1A and V2 receptor antagonist, in vitro and in vivo. $\mathrm{J}$ Pharmacol Exp Ther 1997;282:301-8.

71. Yatsu T, Tomura Y, Tahara A, et al. Cardiovascular and renal effects of conivaptan hydrochloride (YM087), a vasopressin V1A and V2 receptor antagonist, in dogs with pacinginduced congestive heart failure. Eur J Pharmacol 1999;376:239-246.

72. Yatsu T, Tomura Y, Tahara A, et al. Pharmacological profile of YM087, a novel nonpeptide dual vasopressin V1A and V2 receptor antagonist, in dogs. Eur J Pharmacol 1997;321:225-230.

73. Gutkowska J, Jankowski M, Lambert C, et al. Oxytocin releases atrial natriuretic peptide by combining with oxytocin receptors in the heart.Proc Natl Acad Sci USA 1997;94:11704-9.

74. Jankowski M, Hajjar F, Kawas SA, et al. Rat heart: a site of oxytocin production and action. Proc Natl Acad Sci USA 1998;95:14558-63. 